\title{
The Application of 20 inch MCP-PMT In LHAASO-WCDA
}

\section{Xiaohao You, ${ }^{a, *}$ Bo Gao, ${ }^{a}$ MingJun Chen, ${ }^{a}$ HuiCai Li, ${ }^{a}$ Cheng $\mathrm{Liu}^{a}$ and $\mathrm{Kai}_{\mathrm{Li}}{ }^{a}$ on behalf of the LHAASO Collaboration \\ (a complete list of authors can be found at the end of the proceedings)}

${ }^{a}$ Institute of High Energy Physics, 19B Yuquan Road, Shijingshan District, Beijing, China

E-mail: youxiaohao@ihep.ac.cn

In the Large High Altitude Air Shower Observatory (LHAASO), the main physics objective of the Water Cherenkov Detector Array (WCDA) is to survey the gamma-ray sky continuously in the energy range from $100 \mathrm{GeV}$ to PeV. The Water Cherenkov detector array, covering an area of about 78,000 $\mathrm{m}^{2}$ area, is constituted by 3120 detector units divided into 3 separate arrays. In the second and third array are installed 2220 20" PMTs instead of the 8 PMT used in the first $150 \times 150 \mathrm{~m}^{2}$ array. This type of PMT has large sensitive area, high quantum efficiency $(\mathrm{QE})$, and large peak-to-valley $(\mathrm{P} / \mathrm{V})$ ratio for single photoelectron detection. In this work, we will report on the application of 20 inch MCP-PMT at LHAASO-WCDA.

$37^{\text {th }}$ International Cosmic Ray Conference (ICRC 2021)

July 12th - 23rd, 2021

Online - Berlin, Germany

\footnotetext{
${ }^{*}$ Presenter
} 


\section{Introduction}

The Water Cherenkov Detector Array, covering an area of about 78, $000 \mathrm{~m}^{2}$ area, is constituted by 3,120 detector units divided into 3 separate arrays. Every array is a single water pond with $4.5 \mathrm{~m}$ depth. Two of them with an effective area of $150 \times 150 \mathrm{~m}^{2}$ contain 900 detector units each. The third array(WCDA-3) with an area of $300 \times 110 \mathrm{~m}^{2}$ contains 1,320 detector units. Each detector unit is divided in $5 \times 5 \mathrm{~m}^{2}$, separated by black plastic curtains vertically hung in the water to isolate the scattered light. A pair of 8" and 1.5" PMTs in each unit of WCDA-1, while a pair of 20" and 3" PMTs in WCDA-2 and WCDA-3.[1].
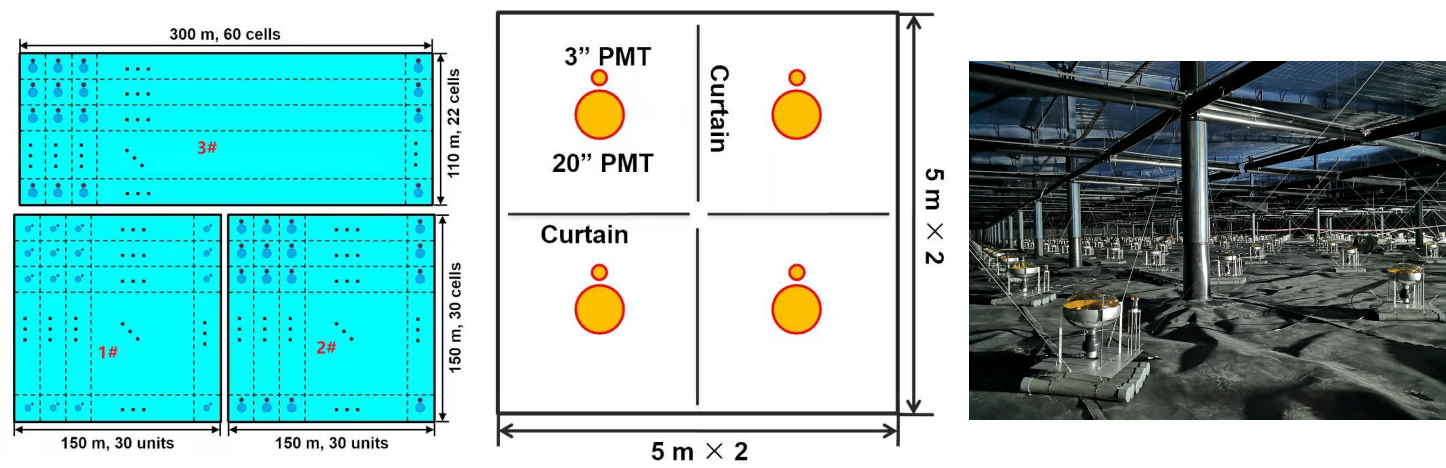

Figure 1: Schematic of LHAASO-WCDA. Left: full picture of the LHAASO WCDA, where are shown the three pools. Middle: Scheme of the arrangement of the PMTs in WCDA-3. Right: a picture of the inside of WCDA-3.

Table 1: Requirements of 20-inch PMTs

\begin{tabular}{l|l|l} 
No. & Item & PMT Specifications \\
1 & Diameter of cathode & 20 in, ellipsoid \\
\hline 2 & Withstand Pressure & $>3 \mathrm{~atm}$ \\
\hline 3 & Working Voltage & Gain@ $5 \times 10^{6},<2000 \mathrm{~V}$ \\
\hline 4 & Working current & $<300 \mu \mathrm{A}$ \\
\hline 5 & Time resolution & $<2 \mathrm{~ns}$ \\
\hline 6 & Peak-to-valley ratio & $>2$ \\
\hline 7 & Response curve & $300 \mathrm{~nm}-650 \mathrm{~nm}$ \\
\hline 8 & TTS & $<7 \mathrm{~ns} @ 5 \times 10^{6}$ \\
\hline 9 & CTTD & $<4 \mathrm{~ns} @ 5 \times 10^{6}$ \\
\hline 10 & Dark noise & $<25 \mathrm{kHz} @ 5 \times 10^{6}$, threshold $=1 / 3 P E, @ T=25^{\circ} \mathrm{C}$ \\
\hline 11 & Nonlinearity & $\geq 1800 P E( \pm 10 \%)$ \\
\hline
\end{tabular}

\section{The 20 inch MCP-PMT}

The 20 inch MCP-PMT is manufactured by North Night Vision Technology Co., Ltd(NNVT) at Nanjing, China. The newly developed 20 inch PMT shown in Fig2, uses micro-channel-plate 
(MCP) instead of the traditional dynodes enabling a better energy resolution and good detector response. It consists of bialkali photocathode, a focusing electrode, a MCP, and an anode. The distance between the photocathode and MCP is nearly $300 \mathrm{~mm}$. Time resolution of the 20-inch MCP-PMT predominantly depends on the electrical field distribution between the photocathode and the MCP, therefore, a lotus-like focusing electrode was designed to reduse transit time spread(TTS) to $5.8 \mathrm{~ns}(\mathrm{FWHM})[2]$.
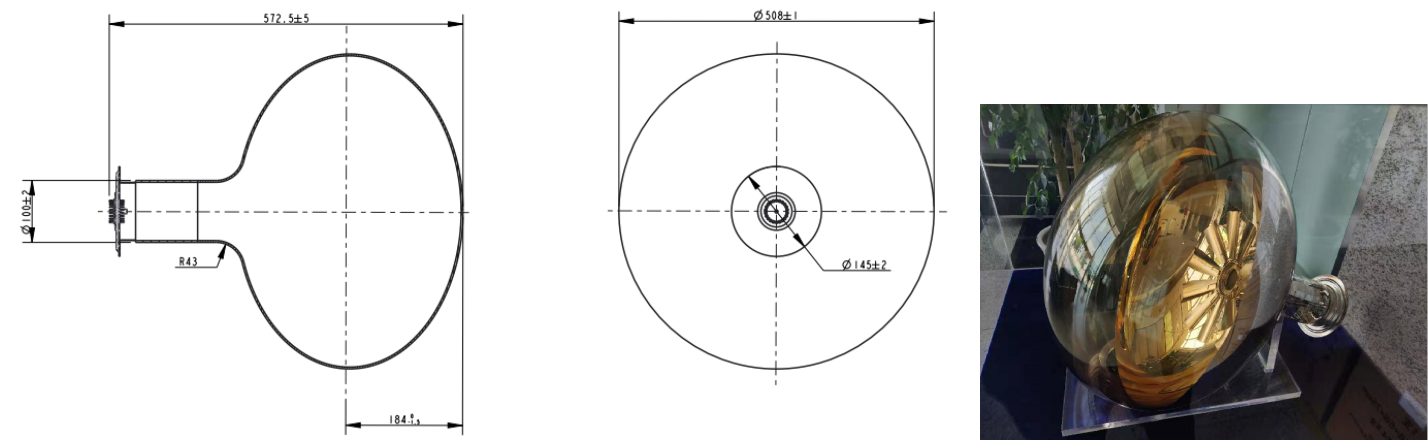

Figure 2: Dimension of 20 inch MCP-PMT.

The average dark noise of the 20 -inch MCP-PMT was $\sim 16 \mathrm{kHz}$ at $\sim 5^{\circ} \mathrm{C}$ in WCDA-3 during dry run mode, and it increased to $\sim 51 \mathrm{kHz}$ once filled with 4.5 meters of water, as shown in Fig. 3.
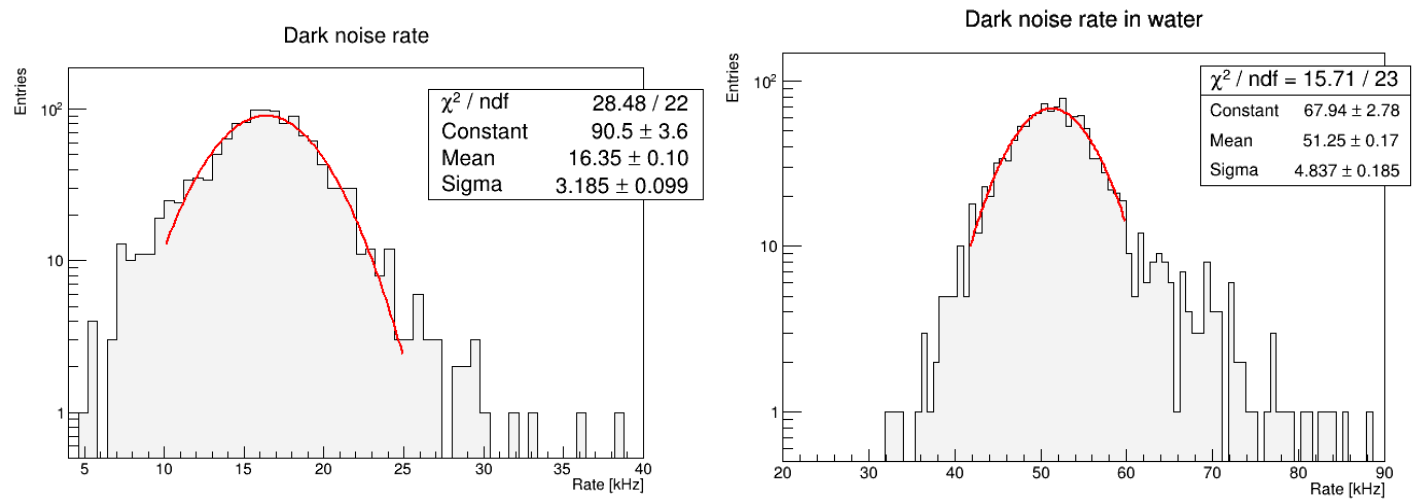

Figure 3: Left: dark noise rate without water, Right: dark noise rate in 4.5 meters of water.

\section{PMT waterproof potting}

The 20 inch MCP-PMTs in LHAASO-WCDA had been anchored at $4.5 \mathrm{~m}$ water depth and will operate at least for 10 years. Waterproof potting of PMT is one of the keys for system reliability. Based from JUNO's ${ }^{1}$ design, waterproof potting was designed to optimize signal quality and stability under water pressure. The potted detector is composed of PMT, HV divider and 30 meters cable (Fig.4(b)). Fig.4(a) show the structure details of waterproof potting.

\footnotetext{
${ }^{1}$ The Jiangmen Underground Neutrino Observatory
} 


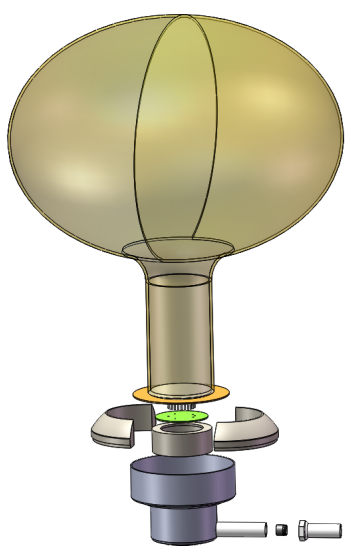

(a) Design of waterproof potting

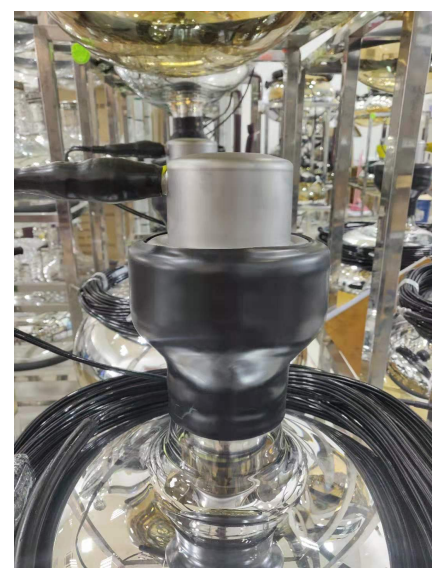

(b) Potted 20 inch PMT

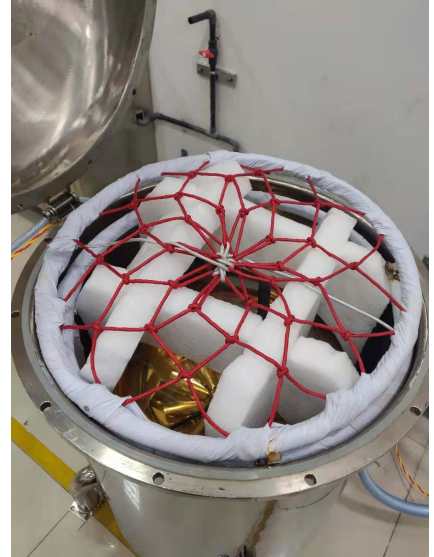

(c) waterproof pressure test

Figure 4: Details of PMT potting.
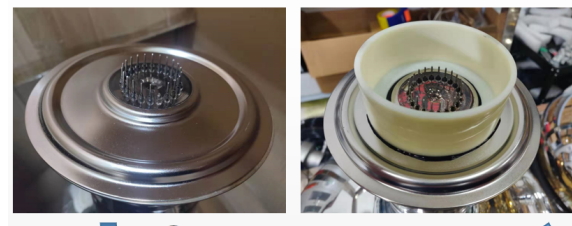

(1)Bonding sleeve made of ABS
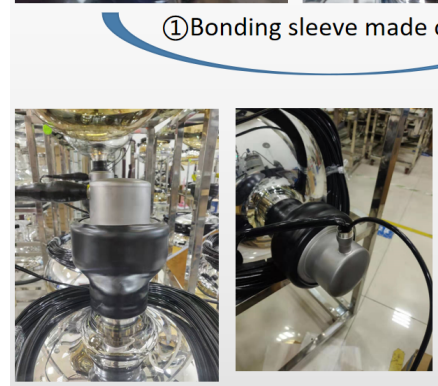

(4) Installing heat shrink tube
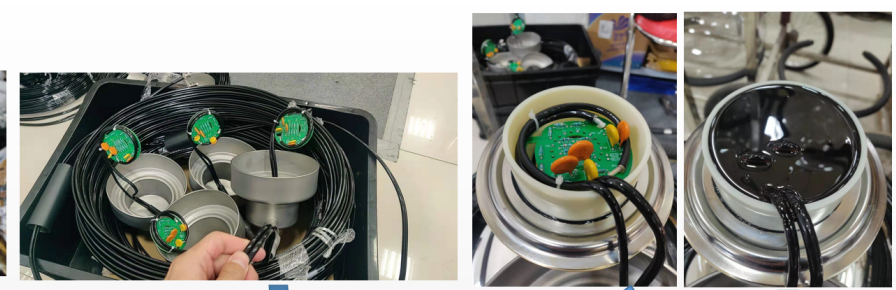

(2) Welding PCB
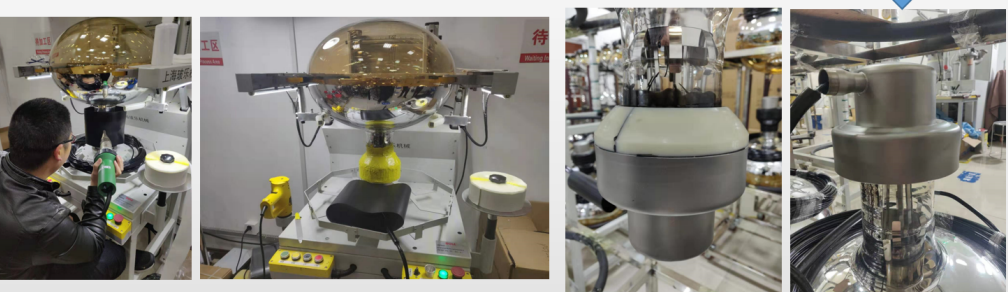

(3)Bongding the shell and injecting glue

Figure 5: PMT potting procedure

Fig.5 shows the complete PMT waterproof potting procedure. To avoid damaging the PMT, the heat shrinkable tube is installed within 2 minutes in step 4.

\section{Test Result of 20 inch MCP-PMT}

Each PMT had been tested at least three times before potting. In order to ensure the potting process didn't damage the PMT, all the 20-inch PMTs will be tested individually again before delivery to LHAASO site. The PMT test system includes two parts: cathode test system and anode test system. 
- The cathode test system is mainly used to test the cathode sensitivity, quantum efficiency, and cathode non-uniformity of the PMT;

- The anode test system is mainly used to test the PMT single photoelectron spectrum, gain, working high voltage, single photoelectron spectrum, peak-to-valley ratio, and energy resolution, such as dark noise rate, transit time dispersion, rise time, fall time, response time, pre-pulse ratio, post-pulse ratio, dynamic range, etc.

All parameters meet LHAASO-WCDA's requirements (Tab 1). Part of test result are shown at Fig 6, where the mean working voltage 1777V(Gain@5 $\left.\times 10^{6}\right)$. The transit time Spread below $7 \mathrm{~ns}$ and the peak-to-Valley ratio greater than 2 .
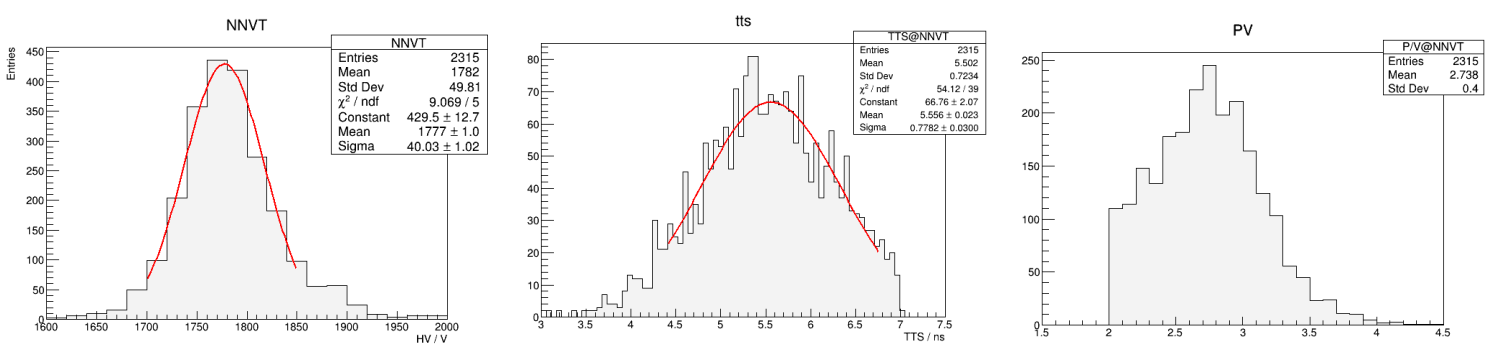

Figure 6: Part of test result at NNVT. Left: the distribution of working voltage, Middle: transit time spread, Right: peak-to-Valley ratio.

\section{The Permalloy Geomagnetic shield}

Due to the large size of 20 inch MCP PMT, the geomagnetic field have big influence on the performance of PMT, including time response, charge resolution and detect efficiency. A Permalloy shielding had been installed on all 20 inch MCP-PMT, as shown at Fig.7. It is a nickel-iron magnetic alloy, with about $80 \%$ nickel and $20 \%$ iron content. Permalloy can change the direction of the magnetic field because of its higher permeability compared to ordinary steel. Since the magnetic field is guided through a low magnetic resistance path, it ensures that the PMT is not affected by the geomagnetic field.

As shown in Fig.8, the time resolution performance of the PMT with magnetic shield is significantly better than that of the PMT without shield.

\section{Summary}

The 20-inch MCP-PMTs are working well at LHAASO-WCDA and the waterproof potting failure rate is less than $1 \%$ as of June 31,2021 . LHASSO-WCDA is taking data and the total array results will be published in October 2021 on current schedule.

\section{Acknowledgements}

The authors would like to thank all staff members who work at the LHAASO site above 4400 meters above sea level year-round to maintain the detector and keep the electrical power supply 

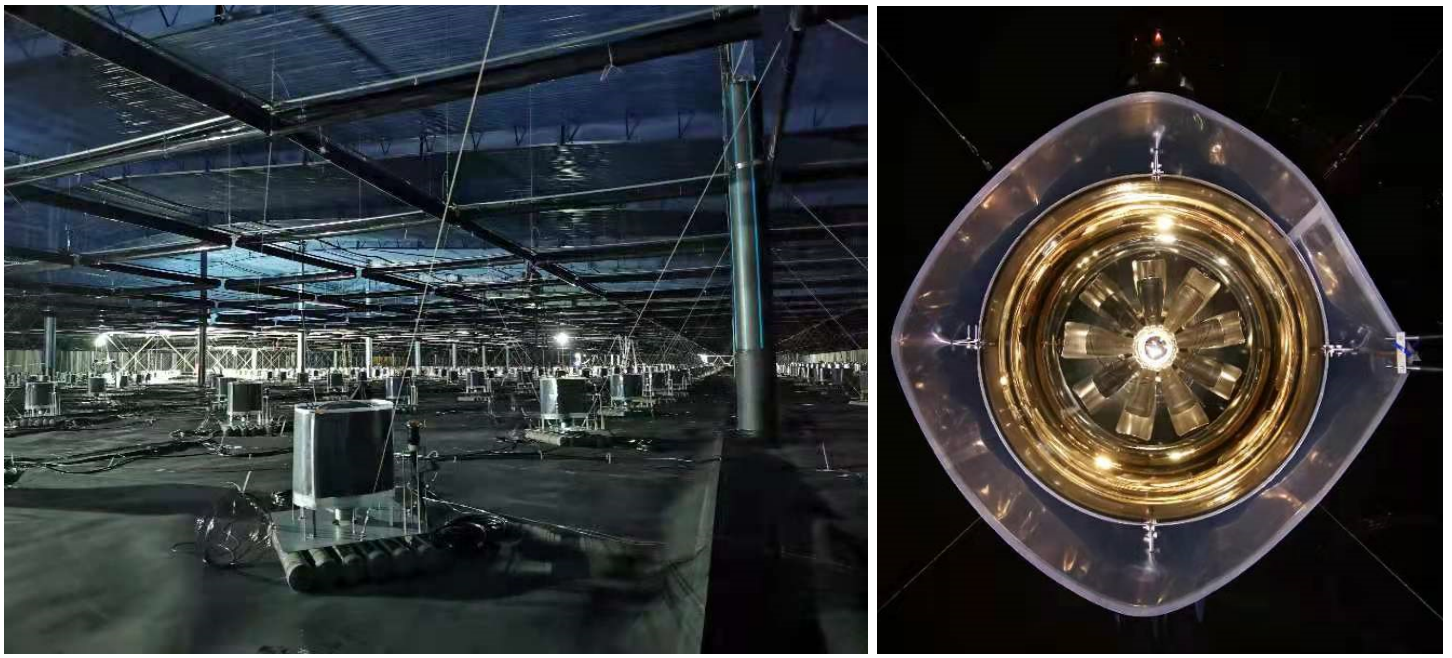

Figure 7: Left: full view of magnetic shield-Permalloy installed on 20 inch MCP-PMT. Right: top view of PMT.
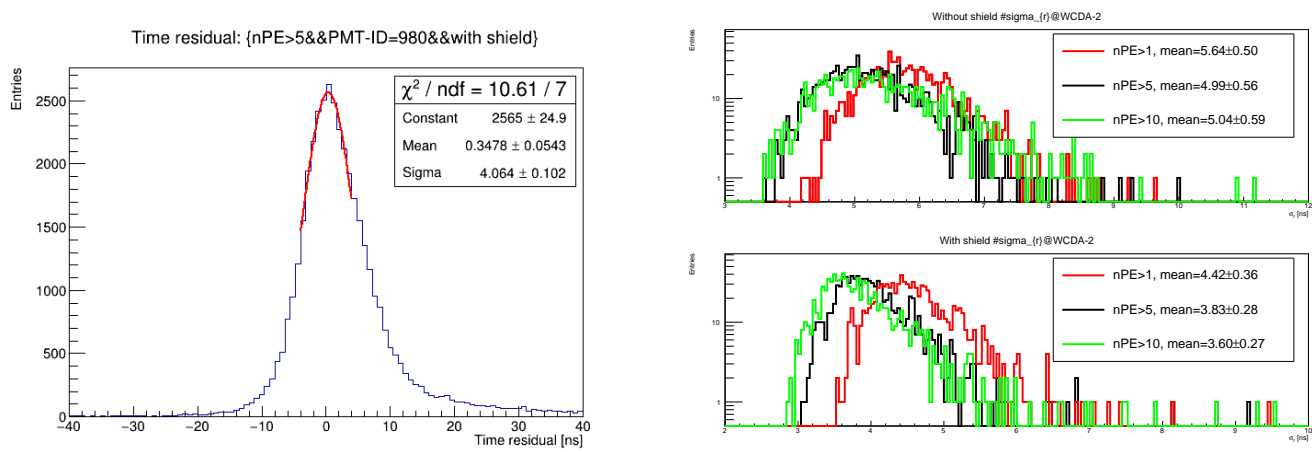

Figure 8: The difference of time residual between PMT with magnetic shield and not. Left: time residual distribution of a PMT with shield when number of photoelectrons(nPE) is great than $5\left(\sigma_{r}=4.08 \mathrm{~ns}\right)$. Right: Comparison of $\sigma_{r}$ of time residual in 900 MCP-PMTs with/without magnetic shield. The red line represents the distribution of $\sigma_{r}$ at nPE $>1\left(E\left(\sigma_{\text {without }- \text { shield }}\right)=5.64 \mathrm{~ns}, E\left(\sigma_{\text {with-shield }}\right)=4.42 \mathrm{~ns}\right)$, the black line represents the distribution of $\sigma_{t}$ at nPE $>5\left(E\left(\sigma_{\text {without }- \text { shield }}\right)=5.00 \mathrm{~ns}, E\left(\sigma_{\text {with-shield }}\right)=3.82 \mathrm{~ns}\right)$, the green line represents the distribution of $\sigma_{r}$ at $\mathrm{nPE}>10\left(E\left(\sigma_{\text {without-shield }}\right)=5.02 \mathrm{~ns}, E\left(\sigma_{\text {with-shield }}\right)=3.60 \mathrm{~ns}\right)$.

and other components of the experiment operating smoothly. We are grateful to the Chengdu Management Committee of Tianfu New Area for their constant financial support of research with LHAASO data.

\section{References}

[1] Cao Zhen, Chen Ming-jun, Chen Song-zhan, Hu Hong-bo, Liu Cheng, Liu Ye, Ma Ling-ling, Ma Xin-hua, Sheng Xiang-dong, Wu Han-rong, Xiao Gang, Yao Zhi-guo, Yin Li-qiao, Zha Min, and Zhang Shou-shan. Introduction to large high altitude air shower observatory. Chinese Astronomy and Astrophysics, 43(4):457-478, 2019. 
[2] Ling Ren, Jianning Sun, Shuguang Si, Xingchao Wang, Muchun Jin, Guorui Huang, Haiyang $\mathrm{Xu}$, Congjie Wang, Wei Hou, Liang Wang, Zhen Jin, Fangjian Qiao, Xiangbiao Qiu, Mengyao Shi, Haoda Zhang, Yiqi Cao, Yan Gu, Shen Li, Xiaoming Han, Sen Qian, and Shulin Liu. Study on the improvement of the 20-inch microchannel plate photomultiplier tubes for neutrino detector. Nuclear Instruments and Methods in Physics Research Section A: Accelerators, Spectrometers, Detectors and Associated Equipment, 977:164333, 2020. 


\section{Full Authors List: LHAASO Collaboration}

Zhen $\mathrm{Cao}^{1,2,3}$, F. Aharonian ${ }^{4,5}$, Q. An ${ }^{6,7}$, Axikegu $^{8}$, L.X. Bai ${ }^{9}$, Y.X. Bai ${ }^{1,3}$, L.X. Bai ${ }^{9}$, Y.X. Bai ${ }^{1,3}$, Y.W. Bao ${ }^{10}$, D. Bastieri ${ }^{11}$, X.J. Bi ${ }^{1,2,3}$, Y.J. Bi ${ }^{1,3}$, H. Cai ${ }^{12}$, J.T. Cai ${ }^{11}$, Zhe Cao ${ }^{6,7}$, J. Chang ${ }^{13}$, J.F. Chang ${ }^{1,3,6}$, B.M. Chen ${ }^{14}$, E.S. Chen ${ }^{1,2,3}$, J. Chen ${ }^{9}$, Liang $\mathrm{Chen}^{1,2,3}$, Liang Chen ${ }^{15}$, Long $\mathrm{Chen}^{8}$, M.J. Chen ${ }^{1,3}$, M.L. Chen ${ }^{1,3,6}$, Q.H. Chen $^{8}$, S.H. Chen ${ }^{1,2,3}$, S.Z. Chen ${ }^{1,3}$, T.L. Chen ${ }^{16}$,X.L. $\mathrm{Chen}^{1,2,3}$, Y. Chen $^{10}$, N. Cheng ${ }^{1,3}$, Y.D. Cheng ${ }^{1,3}$, S.W. Cui ${ }^{14}$, X.H. Cui ${ }^{17}$, Y.D. Cui ${ }^{18}$, B. D’Ettorre Piazzoli ${ }^{19}$, B.Z. Dai ${ }^{20}$, H.L. Dai $^{1,3,6}$, Z.G. Dai ${ }^{7}$, Danzengluobu ${ }^{16}$, D. della Volpe ${ }^{21}$, X.J. Dong ${ }^{1,3}$, K.K. Duan ${ }^{13}$, J.H. Fan ${ }^{11}$, Y.Z. Fan $^{13}$, Z.X. Fan ${ }^{1,3}$, J. Fang ${ }^{20}$, K. Fang $^{1,3}$, C.F. Feng ${ }^{22}$, L. Feng ${ }^{13}$, S.H. Feng ${ }^{1,3}$, Y.L. Feng ${ }^{13}$, B. Gao ${ }^{1,3}$, C.D. Gao ${ }^{22}$, L.Q. Gao ${ }^{1,2,3}$, Q. Gao ${ }^{16}$, W. Gao ${ }^{22}$, M.M. Ge $^{20}$ L.S. Geng ${ }^{1,3}$, G.H. Gong ${ }^{23}$, Q.B. Gou ${ }^{1,3}$, M.H. Gu ${ }^{1,3,6}$, F.L. Guo ${ }^{15}$, J.G. Guo ${ }^{1,2,3}$, X.L. Guo ${ }^{8}$, Y.Q. Guo ${ }^{1,3}$, Y.Y. Guo ${ }^{1,2,3,13}$, Y.A. $\mathrm{Han}^{24}$, H.H. He $\mathrm{H}^{1,2,3}$, H.N. He $\mathrm{H}^{13}$, J.C. He ${ }^{1,2,3}$, S.L. He ${ }^{11}$, X.B. He ${ }^{18}$, Y. He ${ }^{8}$, M. Heller ${ }^{21}$, Y.K. Hor ${ }^{18}$, C. Hou ${ }^{1,3}$, H.B. Hu ${ }^{1,2,3}$, S

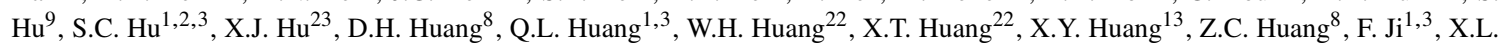
$\mathrm{Ji}^{1,3,6}$, H.Y. Jia ${ }^{8}$, K. Jiang ${ }^{6,7}$, Z.J. Jiang ${ }^{20}$, C. Jin ${ }^{1,2,3}$, T. Ke ${ }^{1,3}$, D. Kuleshov ${ }^{25}$, K. Levochkin ${ }^{25}$, B.B. Li ${ }^{14}$, Cheng Li ${ }^{6,7}$, Cong Li ${ }^{1,3}$,

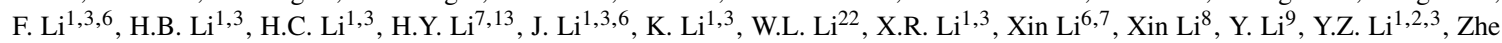

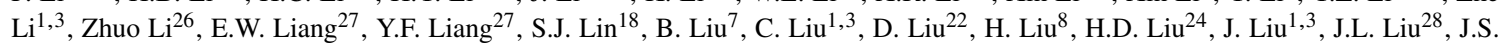

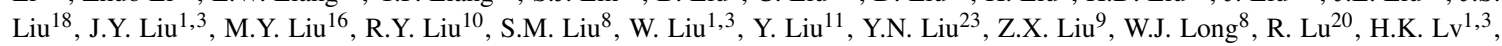

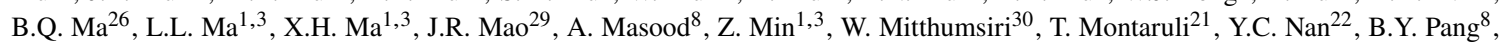
P. Pattarakijwanich ${ }^{30}$, Z.Y. Pei ${ }^{11}$, M.Y. Qi ${ }^{1,3}$, Y.Q. Qi ${ }^{14}$, B.Q. Qiao ${ }^{1,3}$, J.J. Qin ${ }^{7}$, D. Ruffolo ${ }^{30}$, V. Rulev ${ }^{25}$, A. Sáiz ${ }^{30}$, L. Shao ${ }^{14}$, O. Shchegolev $^{25,31}$, X.D. Sheng ${ }^{1,3}$, J.Y. Shi ${ }^{1,3}$, H.C. Song ${ }^{26}$, Yu.V. Stenkin ${ }^{25,31}$, V. Stepanov ${ }^{25}$, Y. Su ${ }^{32}$, Q.N. Sun ${ }^{8}$, X.N. Sun ${ }^{27}$, Z.B.

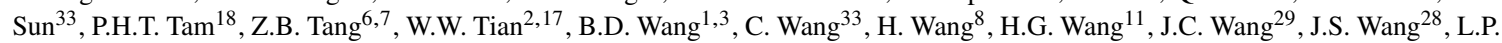
Wang $^{22}$, L.Y. Wang ${ }^{1,3}$, R.N. Wang ${ }^{8}$, W. Wang ${ }^{18}$, W. Wang ${ }^{12}$, X.G. Wang ${ }^{27}$, X.J. Wang ${ }^{1,3}$, X.Y. Wang ${ }^{10}$, Y. Wang ${ }^{8}$, Y.D. Wang ${ }^{1,3}$, Y.J. Wang $^{1,3}$, Y.P. Wang ${ }^{1,2,3}$, Z.H. Wang ${ }^{9}$, Z.X. Wang ${ }^{20}$, Zhen Wang ${ }^{28}$, Zheng Wang ${ }^{1,3,6}{ }^{3}$ D.M. Wei ${ }^{13}$, J.J. Wei ${ }^{13}$, Y.J. Wei ${ }^{1,2,3}$, T. Wen $^{20}$, C.Y. $\mathrm{Wu}^{1,3}$, H.R. $\mathrm{Wu}^{1,3}$, S. $\mathrm{Wu}^{1,3}$, W.X. $\mathrm{Wu}^{8}$, X.F. Wu ${ }^{13}$, S.Q. Xi ${ }^{1,3}$, J. Xia ${ }^{7,13}$, J.J. Xia ${ }^{8}$, G.M. Xiang ${ }^{2,15}$, D.X. Xiao ${ }^{16}$, G. Xiao ${ }^{1,3}$, H.B. Xiao ${ }^{11}$, G.G. Xin ${ }^{12}$, Y.L. Xin ${ }^{8}$, Y. Xing ${ }^{15}$, D.L. Xu ${ }^{28}$, R.X. Xu ${ }^{26}$, L. Xue ${ }^{22}$, D.H. Yann ${ }^{29}$, J.Z. Yan ${ }^{13}$, C.W. Yang 9 , F.F. Yang ${ }^{1,3,6}$, J.Y. Yang ${ }^{18}$, L.L. Yang ${ }^{18}$, M.J. Yang ${ }^{1,3}$, R.Z. Yang ${ }^{7}$, S.B. Yang ${ }^{20}$, Y.H. Yao ${ }^{9}$, Z.G. Yao ${ }^{1,3}$, Y.M. Ye ${ }^{23}$, L.Q. Yin ${ }^{1,3}$, N. Yin ${ }^{22}$, X.H. You $^{1,3}$, Z.Y. You ${ }^{1,2,3}$, Y.H. Yu ${ }^{22}$, Q. Yuan ${ }^{13}$, H.D. Zeng ${ }^{13}$, T.X. Zeng ${ }^{1,3,6}$, W. Zeng ${ }^{20}$, Z.K. Zeng ${ }^{1,2,3}$, M. Zha ${ }^{1,3}$, X.X. Zhai ${ }^{1,3}$, B.B. Zhang $^{10}$, H.M. Zhang ${ }^{10}$, H.Y. Zhang ${ }^{22}$, J.L. Zhang ${ }^{1}$, J.W. Zhang ${ }^{9}$, L.X. Zhang ${ }^{11}$, Li Zhang ${ }^{20}$, Lu Zhang ${ }^{14}$, P.F. Zhang ${ }^{20}$, P.P. Zhang ${ }^{14}$, R. Zhang ${ }^{7,13}$, S.R. Zhang ${ }^{14}$, S.S. Zhang ${ }^{1,3}$, X. Zhang ${ }^{10}$, X.P. Zhang ${ }^{1,3}$, Y.F. Zhang ${ }^{8}$, Y.L. Zhang ${ }^{1,3}$, Yi Zhang ${ }^{1,13}$, Yong Zhang ${ }^{1,3}$, B. Zhao $^{8}$, J. Zhao ${ }^{1,3}$, L. Zhao ${ }^{6,7}$, L.Z. Zhao ${ }^{14}$, S.P. Zhao ${ }^{13,22}$, F. Zheng ${ }^{33}$, Y. Zheng ${ }^{8}$, B. Zhou ${ }^{1,3}$, H. Zhou ${ }^{28}$, J.N. Zhou ${ }^{15}$, P. Zhou ${ }^{10}$, R. Zhou $^{9}$, X.X. Zhou ${ }^{8}$, C.G. Zhu ${ }^{22}$, F.R. Zhu ${ }^{8}$, H. Zhu ${ }^{17}$, K.J. Zhu ${ }^{1,2,3,6}$ and X. Zuo ${ }^{1,3}$

${ }^{1}$ Key Laboratory of Particle Astrophyics \& Experimental Physics Division \& Computing Center, Institute of High Energy Physics, Chinese Academy of Sciences, 100049 Beijing, China

${ }^{2}$ University of Chinese Academy of Sciences, 100049 Beijing, China.

${ }^{3}$ TIANFU Cosmic Ray Research Center, Chengdu, Sichuan, China.

${ }^{4}$ Dublin Institute for Advanced Studies, 31 Fitzwilliam Place, 2 Dublin, Ireland.

${ }^{5}$ Max-Planck-Institut for Nuclear Physics, P.O. Box 103980, 69029 Heidelberg, Germany.

${ }^{6}$ State Key Laboratory of Particle Detection and Electronics, China.

${ }^{7}$ University of Science and Technology of China, 230026 Hefei, Anhui, China.

${ }^{8}$ School of Physical Science and Technology \& School of Information Science and Technology, Southwest Jiaotong University, 610031 Chengdu, Sichuan, China.

${ }^{9}$ College of Physics, Sichuan University, 610065 Chengdu, Sichuan, China

${ }^{10}$ School of Astronomy and Space Science, Nanjing University, 210023 Nanjing, Jiangsu, China.

${ }^{11}$ Center for Astrophysics, Guangzhou University, 510006 Guangzhou, Guangdong, China.

${ }^{12}$ School of Physics and Technology, Wuhan University, 430072 Wuhan, Hubei, China.

${ }^{13}$ Key Laboratory of Dark Matter and Space Astronomy, Purple Mountain Observatory, Chinese Academy of Sciences, 210023 Nanjing, Jiangsu, China.

${ }^{14}$ Hebei Normal University, 050024 Shijiazhuang, Hebei, China.

${ }^{15}$ Key Laboratory for Research in Galaxies and Cosmology, Shanghai Astronomical Observatory, Chinese Academy of Sciences, 200030 Shanghai, China.

${ }^{16}$ Key Laboratory of Cosmic Rays (Tibet University), Ministry of Education, 850000 Lhasa, Tibet, China.

${ }^{17}$ National Astronomical Observatories, Chinese Academy of Sciences, 100101 Beijing, China.

${ }^{18}$ School of Physics and Astronomy \& School of Physics (Guangzhou), Sun Yat-sen University, 519000 Zhuhai, Guangdong, China.

${ }^{19}$ Dipartimento di Fisica dell'Università di Napoli ‘Federico II”, Complesso Universitario di Monte Sant’Angelo, via Cinthia, 80126 Napoli, Italy.

${ }^{20}$ School of Physics and Astronomy, Yunnan University, 650091 Kunming, Yunnan, China.

${ }^{21}$ Departement de Physique Nucleaire et Corpusculaire, Faculte de Sciences, Universite de Gen 'eve, 24 Quai Ernest Ansermet, 1211 Geneva, Switzerland.

${ }^{22}$ Institute of Frontier and Interdisciplinary Science, Shandong University, 266237 Qingdao, Shandong, China.

${ }^{23}$ Department of Engineering Physics, Tsinghua University, 100084 Beijing, China.

${ }^{24}$ School of Physics and Microelectronics, Zhengzhou University, 450001 Zhengzhou, Henan, China. 
${ }^{25}$ Institute for Nuclear Research of Russian Academy of Sciences, 117312 Moscow, Russia.

${ }^{26}$ School of Physics, Peking University, 100871 Beijing, China.

${ }^{27}$ School of Physical Science and Technology, Guangxi University, 530004 Nanning, Guangxi, China.

${ }^{28}$ Tsung-Dao Lee Institute \& School of Physics and Astronomy, Shanghai Jiao Tong University, 200240 Shanghai, China.

${ }^{29}$ Yunnan Observatories, Chinese Academy of Sciences, 650216 Kunming, Yunnan, China.

${ }^{30}$ Department of Physics, Faculty of Science, Mahidol University, 10400 Bangkok, Thailand.

${ }^{31}$ Moscow Institute of Physics and Technology, 141700 Moscow, Russia.

${ }^{32}$ Key Laboratory of Radio Astronomy, Purple Mountain Observatory, Chinese Academy of Sciences, 210023 Nanjing, Jiangsu, China.

${ }^{33}$ National Space Science Center, Chinese Academy of Sciences, 100190 Beijing, China. 